\title{
Dynamic Tsallis Entropy for Simple Model Systems
}

\author{
N.R. Khusnutdinov*, R.M. Yulmetyev \\ AND N.A. EMELYANOVA \\ Department of Theoretical Physics, Kazan State Pedagogical University \\ Mezhlauk 1, 420021, Kazan, Russia \\ (Received September 2, 2005) \\ In this paper we consider the dynamic Tsallis entropy and employ it for \\ four model systems: (i) the motion of Brownian oscillator, (ii) the motion \\ of Brownian oscillator with noise, (iii) the fluctuation of particle density in \\ hydrodynamics limit as well as in (iv) ideal gas. We show that the small \\ value of parameter non-extensivity $0<q<1$ acts as a non-linear magnifier \\ for small values of the entropy. The frequency spectra become more sharp \\ and it is possible to extract useful information in the case of noise. We show \\ that the ideal gas remains non-Markovian for arbitrary values of $q$.
}

PACS numbers: $65.40 . \mathrm{Gr}$

\section{Introduction}

There is a great interest towards studying complex systems of physical, chemical, biological, physiological, and financial origin by using different approaches and concepts $[1,2]$. Significant role in these investigations is played by a concept of information entropy such as Shannon and Renyi entropy, Kolmogorov-Sinai entropy rate [3-7]. In Refs. [8-10] the notion of dynamical information Shannon entropy $^{\dagger}$ has been defined for complex systems. It was suggested to generalize the Shannon entropy by considering square of time correlation function (TCF) as a probability of state. Then the entropy becomes a function of time. The dynamic Shannon entropy has been successfully used to obtain new information in dynamics of $R R$-intervals from human ECG, physiological activity of the individuals, and short-time human memory [8-10]. The investigations reveal also the great role of frequency spectrum of dynamic Shannon entropy.

\footnotetext{
*corresponding author; e-mail: nail@kazan-spu.ru

${ }^{\dagger}$ In literature there is standard notion of dynamic entropy (see e.g. Ref. [11]). We suggest and use the functions $S$ and $S_{q}$ which we call the dynamic Shannon entropy and dynamic Tsallis entropy.
} 
A new approach for entropy was suggested by Tsallis in Ref. [12] (see the earlier papers on this subject in Ref. [13]). This entropy is characterized by one parameter $q$ which was called non-extensive parameter. The natural boundary of this parameter is unity. It means that for $q \rightarrow 1$ the Tsallis entropy transforms to the Shannon entropy. Nevertheless, the cases with $q>0$ and even the limit $q \rightarrow \infty$ were often considered. There is/was huge activity for application of this new notion to various complex systems (see, for example, Ref. [7] and references therein). It was shown that this entropy gives a new kind of distribution. Many complex systems in nature are described by this non-Gaussian distribution with different values of parameter $q$.

The goal of this paper is generalization of dynamic Shannon entropy in the same manner as the Tsallis entropy generalizes the Shannon entropy. In other words, we consider the Tsallis entropy with square of TCF as probability of state. We refer this entropy as dynamic Tsallis entropy (DTE). The real systems in nature have very complicated behavior - the useful signal is lost in "sea" of noises and random unexpected influences. For this reason we would like to consider the dynamic Tsallis entropy for model systems, which nevertheless have deep physical contents. Application of the present theory to the alive systems was considered in Ref. [14]. We employ the information Tsallis entropy and its frequency spectrum with different values of parameter $q$ for model systems. We exploit four well-known models. The first one is the TCF of position of oscillator which performs the Brownian motion. In order to consider more real situation we use the motion of Brownian oscillator with noise in second model. The noise is modelled by generator of random numbers. The third model is the TCF of relaxation of particle density fluctuations in hydrodynamics limit (the Landau-Placzek formula). We consider a specific medium — helium. The fourth model describes the relaxation of particle density fluctuations in ideal gas. In the latter case we additionally calculate the spectrum of non-Markovity parameter for different $q$. The non-Markovity parameter and its spectrum was firstly suggested in Refs. [15, 16] and it was calculated for ideal gas in Ref. [17]. This parameter characterizes the statistical memory effects in systems. In present paper we use slightly different approach and define the non-Markovity parameter in terms of the entropy.

In all considered models the behavior of the dynamic Tsallis entropies and their frequency spectrum crucially depend on the parameter $q$. We observe that the decrease in this parameter $q \rightarrow 0$ allows us to enlarge the fine structure of entropies and to make their frequency spectrum more sharp.

The organization of this paper is as follows. In Sect. 2 we discuss the well known hierarchy of Zwanzig-Mori's kinetic equations and general properties of the DTE and define different kinds of relaxation times. The TCF of Brownian motion of oscillator is considered in Sect. 3 and with noise in Sect. 4 . The formula of Landau and Placzek for relaxation of density fluctuations are exploited in Sect. 5. 
The TCF of density fluctuations in ideal gas is used in Sect. 6. We finish our paper by concluding remarks in Sect. 7 .

\section{The Zwanzig-Mori hierarchy and dynamic Tsallis entropies}

At the beginning we shortly discuss the well known hierarchy of the ZwanzigMori equations $[18,19]$. Let us consider the dynamic variable $\delta A(t)$. It may be, for example, the Fourier component of density fluctuation. This variable obeys the Liouville equation

$$
\frac{\mathrm{d} \delta A(t)}{\mathrm{d} t}=\mathrm{i} \widehat{L} \delta A(t)
$$

Applying $n$ times the Liouville operator $\widehat{L}$ to the initial variable $\delta A(0)$ we obtain the infinite set of variables $B_{n}(0)$ :

$$
B_{n}(0)=\widehat{L}^{n} \delta A(0)
$$

by using which and the Liouville equation we may obtain the initial dynamical variable in arbitrary moment of time

$$
\delta A(t)=\sum_{n=0}^{\infty} \frac{(\mathrm{i} t)^{n}}{n !} B_{n} .
$$

Applying the Gram-Schmidt orthogonalization procedure [20] to this set of functions we obtain the complete set of dynamic variables $W_{n}$ which are orthogonal at the initial time

$$
\left\langle W_{n}^{*} W_{l}\right\rangle=\left\langle\left|W_{n}\right|^{2}\right\rangle \delta_{n, l}
$$

where $\langle\ldots\rangle$ denotes the statistical average over the Gibbs ensemble. If the dynamic variable is evaluated by the Liouville operator, then the orthogonality is preserved at any moment of time due to the self-adjointness of the Liouville operator.

The time correlation function $M_{0}$ of variable $W_{0}=\delta A$ is defined as follows:

$$
M_{0}(t)=\frac{\left\langle W_{0}(0)^{*} W_{0}(t)\right\rangle}{\left\langle\left|W_{0}(0)\right|^{2}\right\rangle}=\frac{\left\langle W_{0}^{*} \exp (\widehat{i} \widehat{L} t) W_{0}\right\rangle}{\left\langle\left|W_{0}\right|^{2}\right\rangle} .
$$

It is well known that this TCF obeys the infinite hierarchy of the Zwanzig-Mori kinetic equations

$$
\frac{\mathrm{d} M_{n}(t)}{\mathrm{d} t}=\mathrm{i} \omega_{0}^{(n)} M_{n}(t)-\Omega_{n+1}^{2} \int_{0}^{t} \mathrm{~d} t^{\prime} M_{n+1}\left(t^{\prime}\right) M_{n}\left(t-t^{\prime}\right)
$$

where

$$
\omega_{0}^{(n)}=\frac{\left\langle W_{n}^{*} \widehat{L} W_{n}\right\rangle}{\left\langle\left|W_{n}\right|^{2}\right\rangle}, \quad \Omega_{n}^{2}=\frac{\left\langle\left|W_{n}\right|^{*}\right\rangle}{\left\langle\left|W_{n-1}\right|^{2}\right\rangle}
$$

and

$$
M_{n}(t)=\frac{\left\langle W_{n}^{*} \exp \left(\mathrm{i} \widehat{L}_{22}^{(n)} t\right) W_{n}\right\rangle}{\left\langle\left|W_{n}\right|^{2}\right\rangle} .
$$


The operator $\widehat{L}_{22}^{(n)}$ is defined in the following manner:

$$
\widehat{L}_{22}^{(n)}=P_{n-1} P_{n-2} \ldots P_{0} \widehat{L} P_{0} \ldots P_{n-2} P_{n-1}
$$

in terms of projectors $P_{n}=1-\Pi_{n}$, where the $\Pi_{n}$ is a projector for state $W_{n}$ :

$$
\Pi_{n}=\frac{\left.W_{n}\right\rangle\left\langle W_{n}^{*}\right.}{\left\langle\left|W_{n}\right|^{2}\right\rangle} .
$$

The functions $M_{n}$ for $n \geq 1$ are not, in fact, usual TCFs, because the operator $\exp \left(\mathrm{i} \widehat{L}_{22}^{(n)} t\right)$ is not an operator of evolution. Nevertheless, we will refer to these functions as TCFs for the next dynamic variables.

The functions $M_{n}$ are considered as functions characterizing the statistical memory of the system. In order to describe quantitatively the non-Markovity of hierarchy, the parameter of non-Markovity and its spectrum were introduced in Refs. $[15,16]$. The spectrum ${ }^{\ddagger}$ of this parameter is defined as ratio of two neighboring relaxation times

$$
\epsilon_{n}=\frac{\tau_{n}}{\tau_{n+1}}
$$

The relaxation time $\tau_{n}$ of function $M_{n}$ was defined as real part of the Laplace image (see Eq. (4)) of this function at zero point

$$
\tau_{n}=\mathcal{R} \widetilde{M}_{n}(0)=\mathcal{R} \int_{0}^{\infty} M_{n}(t) \mathrm{d} t,
$$

where $\mathcal{R}$ means a real part. Because the function $M_{n+1}$ is an integral core of integro-differential equation (1) for $M_{n}$, then this parameter (3) compares the integral core with function itself. More precisely we compare squares under TCFs. If parameter $\epsilon_{n}$ is around unity, this level (level means $n$ ) is non-Markovian: we cannot transform integro-differential equation for $M_{n}$ to differential one, and vice versa, if this parameter tends to infinity, the core has sharp peak and we may transform the integro-differential equation for $M_{n}$ to differential one. In this case there is no memory (integral) in this level. In this paper we use slightly different definition for relaxation time (see Sect. 6).

Applying the Laplace transformation

$$
\widetilde{M}_{n}(s)=\int_{0}^{\infty} \mathrm{d} t \mathrm{e}^{-s t} M_{n}(t)
$$

to Eq. (1), we transform this hierarchy to the infinite system of algebraic equations

$$
\widetilde{M}_{n}(s)=\left[s-\mathrm{i} \omega_{0}^{(n)}+\Omega_{n+1}^{2} \widetilde{M}_{n+1}(s)\right]^{-1},
$$

by using which we may express $M_{n}$ in term of zero TCF $M_{0}$.

In statistical physics of non-equilibrium systems the TCF acts as the function of distribution and pair correlation and can be used to calculate different thermodynamical parameters and the spacial structure of the system [21]. For many physical discrete systems it is impossible to find a distribution function.

\footnotetext{
${ }^{\ddagger}$ This is not frequency spectrum. Here the spectrum means set of parameters $\epsilon_{n}$.
} 
For this reason it seems optimal to obtain the TCF for investigations of complex systems with the help of integro-differential equations which are based on small increments of time and independent variables.

The set of the measured parameters of the complex system may be represented as a set of fluctuations [14]:

$$
Z=\{\zeta(T), \zeta(T+\tau), \zeta(T+2 \tau), \ldots, \zeta(T+k \tau), \ldots, \zeta(T+\tau N-\tau)\},
$$

where $\zeta$ represents the fluctuation $\delta x$ of some quantity $x$.

Let us define the sampling by length $k$, which starts at the moment $T+m \tau$ by the relation

$$
\zeta_{m+k}^{m}=\{\zeta(T+m \tau), \ldots, \zeta[T+m \tau+(k-1) \tau]\} .
$$

The operator which projects the sampling by length $k$ on the sampling at the initial moment of time has the form

$$
\Pi=\frac{\left|\zeta_{k}^{0}\right\rangle\left\langle\zeta_{k}^{0}\right|}{\left\langle\zeta_{k}^{0} \zeta_{k}^{0}\right\rangle}
$$

where angle brackets mean the scalar product (time average). The utilization of this projection operator allows us to represent the sampling as a sum of two independent parts

$$
\zeta_{m+k}^{m}=\zeta_{m+k}^{m}{ }^{\prime}+\zeta_{m+k}^{m}{ }^{\prime \prime},
$$

where

$$
\begin{aligned}
& \zeta_{m+k}^{m}{ }^{\prime}=\Pi \zeta_{m+k}^{m}=\zeta_{k}^{0} M_{0}(t), \\
& \zeta_{m+k}^{m}{ }^{\prime \prime}=(1-\Pi) \zeta_{m+k}^{m}=\zeta_{m+k}^{m}-\zeta_{k}^{0} M_{0}(t),
\end{aligned}
$$

and TCF $M_{0}(t)$ is defined by $(2)$.

It is easy to see that

$$
\left\langle\left(\zeta_{m+k}^{m}\right)^{2}\right\rangle=\left\langle\left(\zeta_{m+k}^{m}\right)^{2}\right\rangle+\left\langle\left(\zeta_{m+k}^{m}\right)^{\prime \prime}\right\rangle
$$

which is the consequence of orthogonality of $(7 a)$ and $(7 b)$.

Direct calculations yield

$$
\left\langle\left(\zeta_{m+k}^{m}\right)^{2}\right\rangle=\left\langle\left(\zeta_{k}^{0}\right)^{2}\right\rangle M_{0}(t)^{2}
$$

For stationary processes, when dispersion does not depend on time, we obtain

$$
\left\langle\left(\zeta_{m+k}^{m}\right)^{\prime \prime}\right\rangle=\left\langle\left(\zeta_{m+k}^{m}\right)^{2}\right\rangle-\left\langle\left(\zeta_{k}^{0}\right)^{2}\right\rangle M_{0}(t)^{2}=\left\langle\left(\zeta_{m+k}^{m}\right)^{2}\right\rangle\left[1-M_{0}(t)^{2}\right] .
$$

Therefore the mean-square value of the fluctuations is presented as a sum of two parts

$$
\left\langle\left(\zeta_{m+k}^{m}\right)^{2}\right\rangle=\left\langle\left(\zeta_{k}^{0}\right)^{2}\right\rangle M_{0}(t)^{2}+\left\langle\left(\zeta_{k}^{0}\right)^{2}\right\rangle\left[1-M_{0}(t)^{2}\right]
$$

or in a generalized form

$$
\left\langle\left(\zeta_{m+k}^{m}\right)^{2}\right\rangle=\left\langle\left(\zeta_{k}^{0}\right)^{2}\right\rangle M_{0}(t)^{2}+\left\langle\left(\zeta_{m+k}^{m}\right)^{2}\right\rangle\left[1-M_{0}(t)^{2}\right] .
$$


The above expression (9) has a standard form of a mean value

$$
\left\langle\left(\zeta_{m+k}^{m}\right)^{2}\right\rangle=\left\langle\left(\zeta_{k}^{0}\right)^{2}\right\rangle P(t)+\left\langle\left(\zeta_{m+k}^{m}\right)^{2}\right\rangle Q(t)
$$

where $P(t)$ is the probability of the state and $Q(t)=1-P(t)$. By analogy with this formula we define

$$
P_{n}(t)=\left|M_{n}(t)\right|^{2}\left(Q_{n}(t)=1-\left|M_{n}(t)\right|^{2}\right), \quad n \geq 0
$$

as the probability of the creation (annihilation) of correlation of fluctuations (or memory) for the $n$-th level of relaxation (see, for details Ref. [17]).

In the recent work of authors [10] the dynamical informational Shannon entropy for the study of complex systems was suggested

$$
\begin{aligned}
S_{n}(t) & =-\sum_{i=c, \mathrm{a}} P_{i}(t) \ln P_{i}(t)=-\left|M_{n}(t)\right|^{2} \ln \left|M_{n}(t)\right|^{2} \\
- & {\left[1-\left|M_{n}(t)\right|^{2}\right] \ln \left[1-\left|M_{n}(t)\right|^{2}\right], }
\end{aligned}
$$

where

$$
\begin{aligned}
& S_{n}^{\mathrm{c}}(t)=-\left|M_{n}(t)\right|^{2} \ln \left|M_{n}(t)\right|^{2}, \\
& S_{n}^{\mathrm{a}}(t)=-\left[1-\left|M_{n}(t)\right|^{2}\right] \ln \left[1-\left|M_{n}(t)\right|^{2}\right] .
\end{aligned}
$$

Here the $S_{n}^{\mathrm{c}}(t)$ is the entropy for the stochastic channels of memory creation, and $S_{n}^{\mathrm{a}}(t)$ is entropy for the stochastic channels of memory annihilation.

The infinite set of TCFs $M_{n}(t)$ produces the infinite set of entropies which are defined by relations

$$
S^{n}[t]=S\left[M_{n}(t)\right], \quad S^{n c}[t]=S^{\mathrm{c}}\left[M_{n}(t)\right], \quad S^{n a}[t]=S^{\mathrm{a}}\left[M_{n}(t)\right] .
$$

Because the TCFs are always smaller than unity, all of these entropies are positive.

We also generalize our definitions of entropy due to Tsallis by introducing parameter of non-extensivity $q$ :

$$
\begin{aligned}
& S_{q}(t)=-\frac{\left[1-\left|M_{0}(t)\right|^{2}\right]^{q}-1+\left|M_{0}(t)\right|^{2 q}}{q-1}, \\
& S_{q}^{\mathrm{c}}(t)=-\frac{\left|M_{0}(t)\right|^{2 q}-\left|M_{0}(t)\right|^{2}}{q-1}, \\
& S_{q}^{\mathrm{a}}(t)=-\frac{\left[1-\left|M_{0}(t)\right|^{2}\right]^{q}-1+\left|M_{0}(t)\right|^{2}}{q-1} .
\end{aligned}
$$

The infinite set entropies are defined by relations

$$
S_{q}^{n}[t]=S_{q}\left[M_{n}(t)\right], \quad S_{q}^{n \mathrm{c}}[t]=S_{q}^{\mathrm{c}}\left[M_{n}(t)\right], \quad S_{q}^{n \mathrm{a}}[t]=S_{q}^{\mathrm{a}}\left[M_{n}(t)\right] .
$$

We also calculate the frequency spectra $\widehat{S}^{n}[\nu], \widehat{S}^{n \mathrm{c}}[\nu], \widehat{S}^{n \mathrm{a}}[\nu], \widehat{S}_{q}^{n}[\nu], \widehat{S}_{q}^{n \mathrm{c}}[\nu], \widehat{S}_{q}^{n \mathrm{a}}[\nu]$ 
of these functions and define the spectra of the relaxation times by relations

$$
\begin{aligned}
& \tau_{n}=\widehat{S}^{n}[\nu]_{\nu=0}, \quad \tau_{n \mathrm{c}}=\widehat{S}^{n \mathrm{c}}[\nu]_{\nu=0}, \quad \tau_{n \mathrm{a}}=\widehat{S}^{n \mathrm{a}}[\nu]_{\nu=0}, \\
& \tau_{q n}=\widehat{S}_{q}^{n}[\nu]_{\nu=0}, \quad \tau_{q n \mathrm{c}}=\widehat{S}_{q}^{n \mathrm{c}}[\nu]_{\nu=0}, \quad \tau_{q n \mathrm{a}}=\widehat{S}_{q}^{n \mathrm{a}}[\nu]_{\nu=0} .
\end{aligned}
$$

We define the frequency spectrum as the Fourier transformation of these quantities by relation

$$
\widehat{M}_{n}(\nu)=\int_{-\infty}^{+\infty} M_{n}(\tau) \mathrm{e}^{-2 \pi \mathrm{i} \nu \tau} \mathrm{d} \tau .
$$

To calculate the Fourier transformation of all quantities we use package for numerical Fourier transformation from package "Mathematica". We tabulate functions $M_{n}(\tau)$ with step $\Delta=1 / 40$ in range $\tau=(0,60)$. The number of points $N=2401$. Then the Fourier transform

$$
\begin{gathered}
\widehat{M}_{n}(\nu)=\int_{-\infty}^{+\infty} M_{n}(\tau) \mathrm{e}^{-2 \mathrm{i} \pi \nu \tau} \mathrm{d} \tau=2 \int_{0}^{+\infty} M_{n}(\tau) \cos (2 \pi \nu \tau) \mathrm{d} \tau \\
\quad=2 \Delta \mathcal{R} \sum_{k=0}^{N-1} M_{n}(k \Delta) \mathrm{e}^{-2 \pi \mathrm{i} k \nu / N}-M_{n}(0)
\end{gathered}
$$

is represented as a function of discrete frequencies $\nu=k / N \Delta(k=0, \ldots, N-1)$

$$
\widehat{M}_{n}(\nu)=2 \Delta \mathcal{R} \sum_{l=0}^{N-1} M_{n}(k \Delta) \mathrm{e}^{-2 \pi \mathrm{i} l k / N}-M_{n}(0) .
$$

Let us summarize here some analytical results. In the limit $q \rightarrow 1$ the formulae (13) transform to Eqs. (12). For this reason we may refer to entropies (12) as entropies (13) at the point $q=1$. The entropy $S_{q}^{n}(13)$ amounts to maximum value, $\left(2^{1-q}-1\right) /(1-q)$, at the point $\left|M_{n}(t)\right|^{2}=\frac{1}{2}$. For small values of $\left|M_{n}(t)\right|^{2}$ the dependence of entropies $S_{q}^{n}, S_{q}^{n \text { c }}$ changes drastically on value of $q$. For $q \ll 1$ we have

$$
S_{q} \approx\left|M_{0}(t)\right|^{2 q}, \quad S_{q}^{\mathrm{c}} \approx\left|M_{0}(t)\right|^{2 q}, \quad S_{q}^{\mathrm{a}} \approx\left|M_{0}(t)\right|^{2}
$$

These expressions are valid for all cases: $\left|M_{0}(t)\right|^{2} \ll q,\left|M_{0}(t)\right|^{2} \gg q$ and $\left|M_{0}(t)\right|^{2} \approx q$. For comparison we have for small values of $\left|M_{0}(t)\right|^{2}: S \approx$ $-\left|M_{0}(t)\right|^{2} \ln \left|M_{0}(t)\right|^{2}$. Therefore $S_{q}^{\mathrm{a}} \ll S_{q}^{\mathrm{c}}, S_{q}, S$ and we observe that the decrease in $q$ acts as a magnifier for small values of $\left|M_{0}(t)\right|^{2}$.

For $q \gg 1$ and still $\left|M_{0}(t)\right|^{2} \ll 1$ we have to consider three cases $q\left|M_{0}(t)\right|^{2} \ll 1, q\left|M_{0}(t)\right|^{2} \approx 1$ and $q\left|M_{0}(t)\right|^{2} \gg 1$. We have correspondingly

$$
\begin{aligned}
S_{q} & \approx\left|M_{0}(t)\right|^{2}, \quad S_{q}^{\mathrm{c}} \approx \frac{1}{q}\left|M_{0}(t)\right|^{2}, \quad S_{q}^{\mathrm{a}} \approx\left|M_{0}(t)\right|^{2}, \quad\left[q\left|M_{0}(t)\right|^{2} \ll 1\right], \\
S_{q} & \approx-\frac{1}{q}\left[\left(1-\left|M_{0}(t)\right|^{2}\right)^{q}-1\right], \quad S_{q}^{\mathrm{c}} \approx \frac{1}{q}\left|M_{0}(t)\right|^{2}, \\
S_{q}^{\mathrm{a}} & \approx-\frac{1}{q}\left[\left(1-\left|M_{0}(t)\right|^{2}\right)^{q}-1\right], \quad\left[q\left|M_{0}(t)\right|^{2} \approx 1\right], \\
S_{q} & \approx \frac{1}{q}, \quad S_{q}^{\mathrm{c}} \approx \frac{1}{q}\left|M_{0}(t)\right|^{2}, \quad S_{q}^{\mathrm{a}} \approx \frac{1}{q}, \quad\left[q\left|M_{0}(t)\right|^{2} \gg 1\right] .
\end{aligned}
$$


We observe that for sufficiently large $q$ and small $\left|M_{0}(t)\right|^{2} \ll 1$ we have: $S_{q}, S_{q}^{\mathrm{c}}, S_{q}^{\mathrm{a}} \ll S$. Therefore we observe that increase in $q$ is a demagnification lens for small values of $\left|M_{0}(t)\right|$. We note that the entropies are equal to zero for arbitrary $q$ for zero value of $M_{0}(t)$ as well as for $M_{0}(t)=1$. It is easy to see that if the TCF $M_{0}(t)$ possesses an extremum at some time $t_{0}$, the entropies have extremum, too. The structure of extrema of TCF generates the same structure of extrema of entropies. After this general frameworks let us consider specific models.

\section{The motion of Brownian oscillator}

Let us consider the Brownian oscillator which models the particle with internal oscillatory degree of freedom with frequency $\omega_{0}$. The friction coefficient $\beta$ describes the movement of the particle in a medium. We may use the general theory of Zwanzig and Mori [18, 19] for this particle because its position and velocity are random quantities.

The position and velocity of the Brownian oscillator is subject for equations

$$
\begin{aligned}
\frac{\mathrm{d} x}{\mathrm{~d} t} & =v, \\
\frac{\mathrm{d} v}{\mathrm{~d} t} & =-2 c v-\omega_{0}^{2} x+\frac{F(t)+f(t)}{m},
\end{aligned}
$$

where $c=\beta / 2 m, F(t)$ - external force, $f(t)$ - the Langevin random force. The random quantities $x, v, f$ are described by the following average values:

$$
\begin{aligned}
& \langle x\rangle=0, \quad\langle v\rangle=0, \quad\langle f\rangle=0, \quad\left\langle x^{2}\right\rangle=\frac{T}{m \omega_{0}^{2}}, \quad\left\langle v^{2}\right\rangle=\frac{T}{m}, \quad\langle x v\rangle=0, \\
& \langle x f\rangle=0, \quad\langle v f\rangle=0, \quad\left\langle f(t) f\left(t^{\prime}\right)\right\rangle=2 T \beta \delta\left(t-t^{\prime}\right),
\end{aligned}
$$

where $T$ - temperature in the units of the energy, $m$ - the mass of Brownian particle.

We use the fluctuation of particle's position $x$ :

$$
W_{0}=x(0)
$$

as the initial dynamic variable. The next orthogonal dynamic variables are calculated with the help of the following recurrent relations:

$$
\begin{aligned}
& W_{1}=\left[\mathrm{i} \hat{L}-\omega_{0}^{(0)}\right] W_{0}, \\
& W_{n}=\left[\mathrm{i} \hat{L}-\omega_{0}^{(n-1)}\right] W_{n-1}+\Omega_{n-1}^{2} W_{n-2}, \quad n>1,
\end{aligned}
$$

where the frequencies $\omega_{0}^{(n)}$ and $\Omega_{n}$ are defined by equations

$$
\omega_{0}^{(n)}=\frac{\left\langle W_{n}^{*} \mathrm{i} \hat{L} W_{n}\right\rangle}{\left\langle\left|W_{n}\right|^{2}\right\rangle}, \quad \Omega_{n}^{2}=\frac{\left\langle\left|W_{n}\right|^{2}\right\rangle}{\left\langle\left|W_{n-1}\right|^{2}\right\rangle} .
$$

The straightforward calculations give

$$
\omega_{0}^{(0)}=\frac{\langle x(0) v(0)\rangle}{\left\langle|x(0)|^{2}\right\rangle}=0, \quad W_{1}=\left(\mathrm{i} \hat{L}-\omega_{0}^{(0)}\right) W_{0}=v(0),
$$




$$
\omega_{0}^{(1)}=\frac{\left\langle v(0) \frac{\mathrm{d} v(0)}{\mathrm{d} t}\right\rangle}{\left\langle|v(0)|^{2}\right\rangle}=-2 c, \quad \Omega_{1}^{2}=\frac{\left\langle|v(0)|^{2}\right\rangle}{\left\langle|x(0)|^{2}\right\rangle}=\omega_{0}^{2}, \quad W_{2}=\frac{f(0)}{m} .
$$

Taking into account the equations of motion (17), one obtains the TCF

$$
M_{0}(t)=\frac{\langle x(t) x(0)\rangle}{\left\langle|x(0)|^{2}\right\rangle}=\frac{r_{-} \mathrm{e}^{r_{+} t}-r_{+} \mathrm{e}^{r_{-} t}}{r_{-}-r_{+}},
$$

where $r_{ \pm}=-\left(c \pm \sqrt{c^{2}-\omega_{0}^{2}}\right)$.

To find the TCF for next levels we exploit the Zwanzig-Mori infinite chain of equations given by Eq. (1). The Laplace image of initial TCF has the following form:

$$
\widetilde{M}_{0}(s)=\frac{s-\left(r_{-}+r_{+}\right)}{\left(s-r_{-}\right)\left(s-r_{+}\right)} .
$$

With $n=0$ we obtain from chain (5) the Laplace image of first memory function

$$
\widetilde{M}_{1}(s)=\frac{1}{s-\left(r_{-}+r_{+}\right)} .
$$

Taking the inverse Laplace transform we obtain the first memory function

$$
M_{1}(t)=\mathrm{e}^{\left(r_{-}+r_{+}\right) t}=\mathrm{e}^{-2 c t} .
$$

The calculation in closed form of the memory functions on the next relaxation levels requires the following detailed elaboration of the structure of the Langevin force $f$. For example, in order to calculate the memory function $M_{2}(t)$ we need frequency $\Omega_{2}$ which may be found by setting the mean value of square of the Langevin force. For this reason we restrict our consideration to first two levels of relaxation.

We consider the TCF of Brownian oscillator in the case of small damping $p=c / \omega_{0} \ll 1$. In this case the TCF $M_{0}(t)$ has the following form:

$$
M_{0}(t)=\frac{\langle x(t) x(0)\rangle}{\langle x(0) x(0)\rangle}=\cos \left(2 \pi \nu^{\prime} t\right) \mathrm{e}^{-c|t|}
$$

and describes the motion of Brownian oscillator with frequency $\omega=2 \pi \nu^{\prime} \gg$ $c=\beta / 2 m$, where $m$ is the mass of oscillator, and $\beta$ is the friction coefficient of Brownian particle [22].

There are two parameters $\nu^{\prime}$ and $c$ which characterize the frequency oscillation and relaxation damping of TCF, respectively. For simplicity we consider dimensionless time $t$, frequency of oscillation $\nu^{\prime}$, and damping parameter $c$. The Fourier transform of square of this TCF has the following form:

$$
\widehat{M_{0}^{2}}(\nu)=\frac{c}{2\left(c^{2}+\pi^{2} \nu^{2}\right)}+\frac{c}{4\left[c^{2}+\pi^{2}\left(\nu-2 \nu^{\prime}\right)^{2}\right]}+\frac{c}{4\left[c^{2}+\pi^{2}\left(\nu+2 \nu^{\prime}\right)^{2}\right]} .
$$

We consider frequency spectrum of square of TCF because the entropies are expressed in terms of square of TCF. Therefore there are three maxima at $\nu=0, \nu= \pm 2 \nu^{\prime}$. In the limit of zero damping $c \rightarrow 0$ we obtain 


$$
\widehat{M_{0}^{2}}(\nu)=\frac{1}{2} \delta(\nu)+\frac{1}{4} \delta\left(\nu-2 \nu^{\prime}\right)+\frac{1}{4} \delta\left(\nu+2 \nu^{\prime}\right)
$$

by using well-known formula

$$
\pi \delta(x)=\lim _{\varepsilon \rightarrow 0} \frac{\varepsilon}{x^{2}+\varepsilon^{2}} .
$$

In this case the spectrum consists of three lines at $\nu=0, \nu= \pm 2 \nu^{\prime}$.

In Fig. 1 we reproduce the entropies (12) and in Fig. 2 we show the spectra of them for $\nu^{\prime}=0.1$ and for $c=0,0.01,0.1,1$. There is a peak in spectra at double frequency $\nu=2 \nu^{\prime}=0.2$ for arbitrary small but non-zero damping $c$. For zero damping $c=0$ this peak disappears in total entropy. There are peaks in $\widehat{S}^{\mathrm{c}}$ and $\widehat{S}^{\mathrm{a}}$ at $\nu=0.2$ but with opposite sign. We note that the increase in the damping leads to smearing the fine structure of entropies.

Calculation of the entropies (13) shows that the small values of $q$ "work" as non-linear magnifier for small value of $M_{0}^{2}$. To illustrate this fact we reproduce in Fig. 3 the entropies (13) and (12) for $q=1,0.1, \nu^{\prime}=0.1, c=0.1$. First of all, as expected from Eq. (15) the smaller entropy the greater magnification. Second,

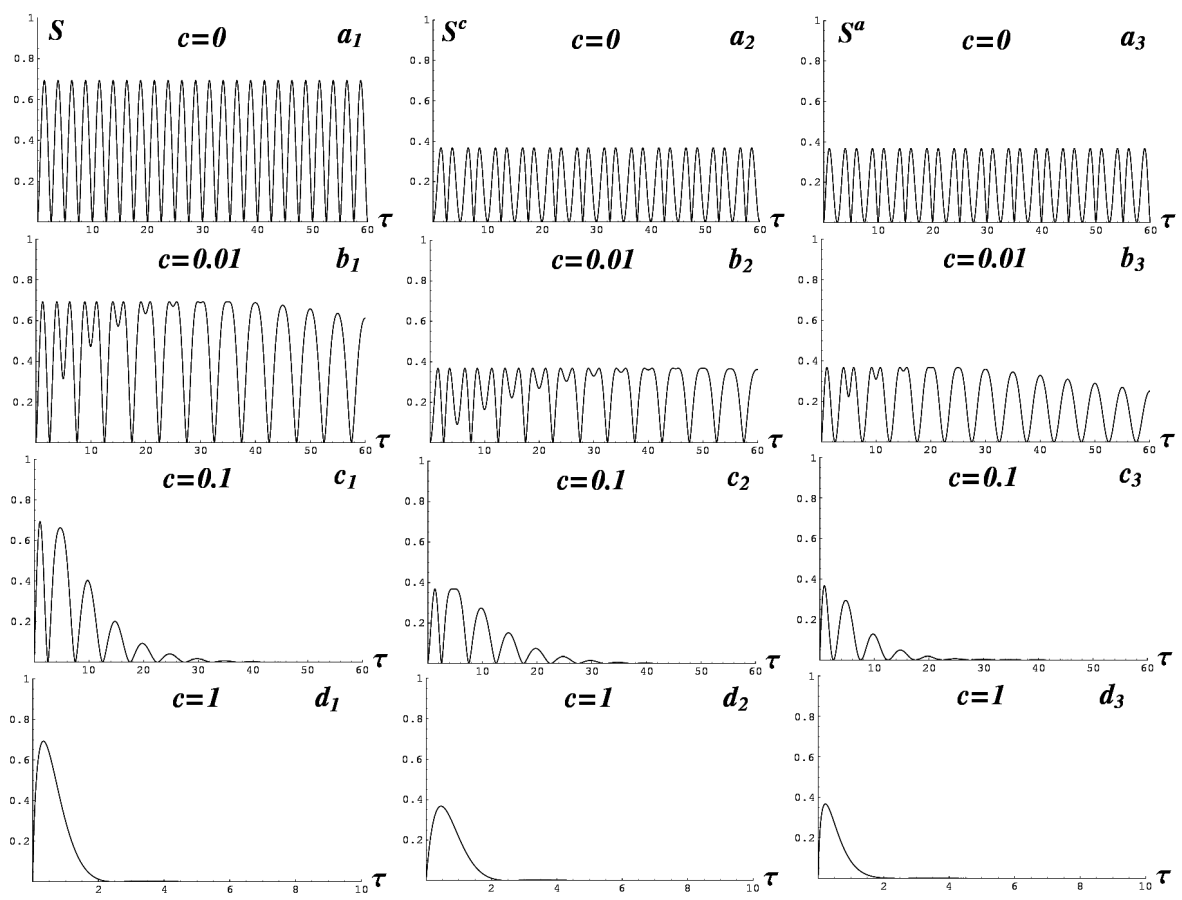

Fig. 1. The plots of entropies (12) for frequency $\nu^{\prime}=0.1$ and $c=0$ (a), 0.01 (b), 0.1 (c), 1 (d) ( $\nu^{\prime}$ and $c$ are oscillation and damping parameters of TCF (21)). It is seen that the time behavior of total $S$ and single channels $S^{\mathrm{c}}$ and $S^{\mathrm{a}}$ of DTEs for various damping regimes reveals a stochastic ordering of time correlation. 


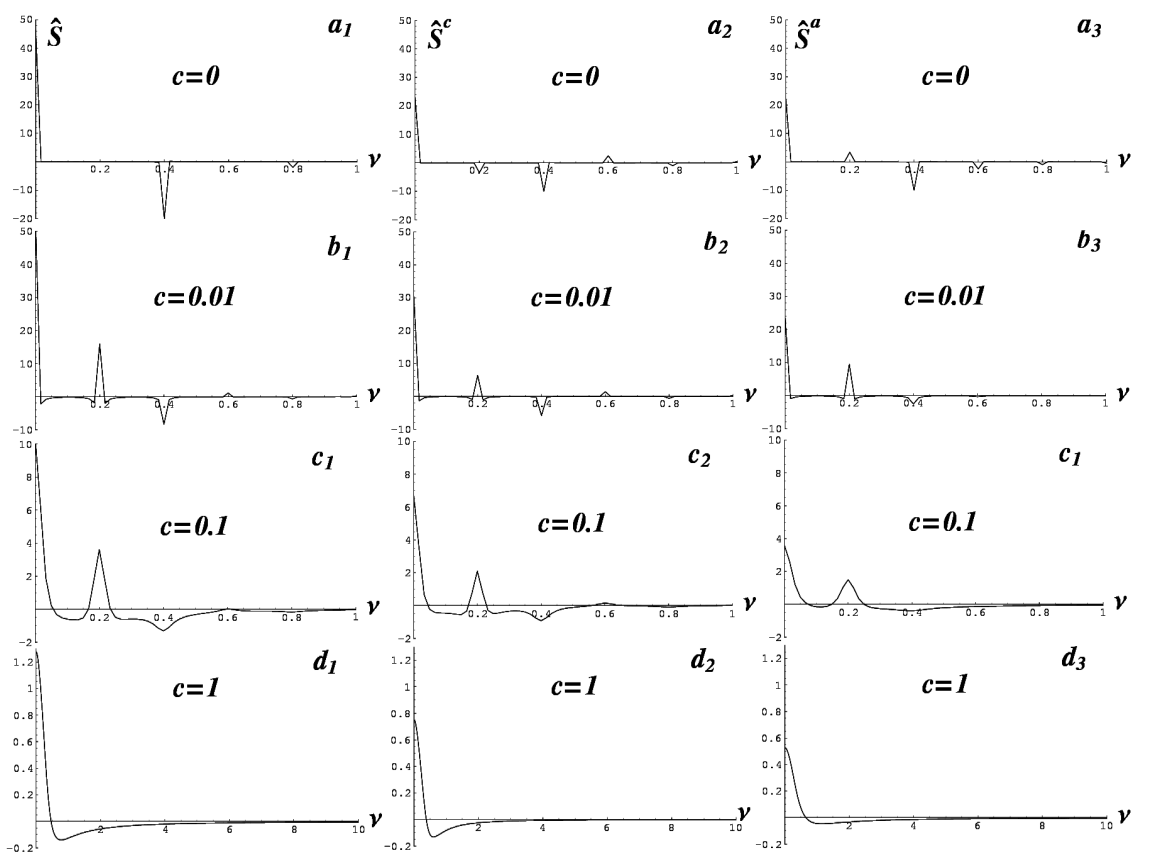

Fig. 2. The plots of spectra of entropies (12) for $\nu^{\prime}=0.1$ and $c=0$ (a), 0.01 (b), 0.1 (c), 1 (d). One can see that a stochastic ordering of time correlation in previous Fig. 1 reduces to an appearance of specific peculiarities in low frequency region. Weak damping $(c=0.1)$ results in amplification of characteristic frequency peaks at $\nu=0.2$ and $\nu=0.4$ whereas zero damping $(c=0)$ leads to a disappearance of specific frequency peaks.

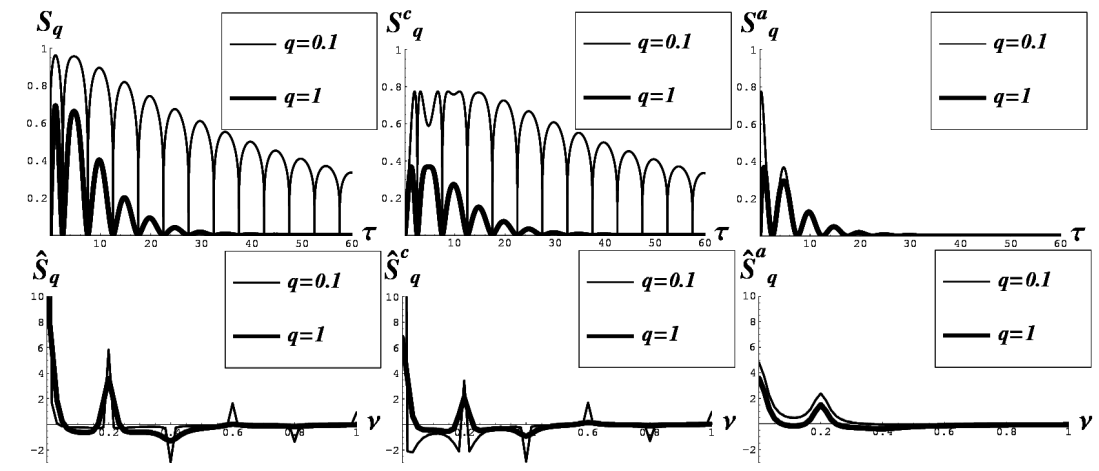

Fig. 3. The plots of entropies (13) for $q=0.1$ and $(12)(q=1)$ and their spectra for $\nu^{\prime}=0.1, c=0.1$. Comparison of dynamic Shannon $(q=1)$ and Tsallis $(q=0.1)$ entropies shows the amplification of DTE for small values of TCF. Therefore one can conclude that DTE acts as a magnifier for small values of TCF. 


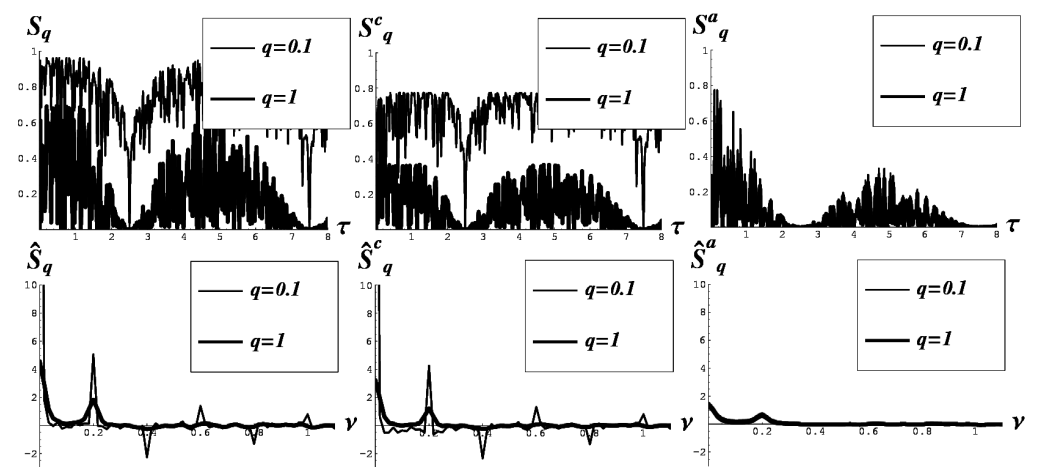

Fig. 4. The plots of entropies (13) for $q=0.1$ and $(12)(q=1)$ and their spectra for $\nu^{\prime}=0.1, c=0.1$ and for Brownian oscillator with noise. Comparison of dynamic Shannon $(q=1)$ and Tsallis $(q=0.1)$ entropies shows the amplification of DTE for small values of TCF. Therefore one can conclude that DTE acts as a magnifier for small values of TCF.

the entropy $S_{q}^{\mathrm{a}}$ does not change sufficiently. The great variation proves $S_{q}$ and $S_{q}^{\text {c }}$. The decrease in $q$ leads to increase in the value of peaks for great frequencies and makes peaks more sharp. The small $q$ makes better sharpness of frequency spectra. This is not the case for $S_{q}^{\mathrm{a}}$. It does not change great.

\section{The motion of Brownian oscillator with noise}

The real signal from alive systems often contains noise (see Ref. [14]). For this reason we suggest the model of Brownian oscillator with noise. We consider the following model of TCF:

$$
M_{0}(t)=R(t) \cos \left(2 \pi \nu^{\prime} t\right) \mathrm{e}^{-c|t|},
$$

where $R(t)$ denotes the random numbers in interval $(-1,1)$ and $R(0)=1$. Therefore the function $R(t)$ makes random the amplitude of oscillation but this is not the case for frequency and damping parameters. At the beginning we know the frequency of oscillations. The TCF has more complicated form which is more close to real dates. The random numbers describe a noise which usually appears in experiment. The time dependence of entropy is much more complicated but nevertheless in this case the DTE works better. We observe the appearing peaks in places, in which we know the peaks must be, but they disappear in noise. There is another observation: the noise is better for frequency spectrum. The peaks for small frequencies look better (see Fig. 4).

\section{Density fluctuations in hydrodynamical limit}

The TCF of density fluctuations in hydrodynamical limit was calculated by Landau and Placzek [22]. It describes scattering of light in liquid in hydrodynamics limit when $k \rightarrow 0$. The TCF has the following form: 


$$
M_{0}(t)=\alpha \mathrm{e}^{-\gamma^{\prime} k^{2} t} \cos \vartheta_{s} k t+(1-\alpha) \mathrm{e}^{-\sigma^{\prime} k^{2} t},
$$

where $\gamma^{\prime}=\frac{1}{2 \rho}\left[\frac{4}{3} \eta+\zeta+\kappa\left(\frac{1}{c_{v}}-\frac{1}{c_{p}}\right)\right], \quad \sigma^{\prime}=\kappa / \rho c_{p}, \alpha=c_{v} / c_{p}$. Here the $c_{v}, c_{p}, \kappa, \eta, \zeta, \rho, \vartheta_{s}$ are specific heat capacities in units of mass at constant volume and constant pressure, the coefficient of thermal conductivity, the coefficient of shear viscosity and volume viscosity, the mass density and sound velocity, correspondingly.

The spectrum of this TCF contains three peaks. The central Rayleigh peak at zero frequency describes isothermal propagation of sound. Two symmetric peaks at frequencies $\omega= \pm \vartheta_{s} k$ describe adiabatic propagation of sound with damping (the Brillouin doublet).

It is more suitable to define dimensionless time $\tau$ by relation $\tau=\vartheta_{s} k t / 2 \pi$. Then the position of the Brillouin doublet will be at the dimensionless frequency $\nu=\omega / 2 \pi=1$, and TCF will take the following form:

$$
M_{0}(\tau)=\alpha \mathrm{e}^{-\gamma k 2 \pi \tau} \cos 2 \pi \tau+(1-\alpha) \mathrm{e}^{-\sigma k 2 \pi \tau},
$$

where $\gamma=\frac{1}{2 \rho \vartheta_{s}}\left[\frac{4}{3} \eta+\zeta+\kappa\left(\frac{1}{c_{v}}-\frac{1}{c_{p}}\right)\right], \sigma=\kappa / \rho c_{p} \vartheta_{s}, \alpha=c_{v} / c_{p}$. We consider a specific medium - helium at temperature $T=20^{\circ} \mathrm{C}$ and pressure $p=1$ bar. In this case we have [23]: $\alpha \approx 0.56, \vartheta_{s} \approx 272 \mathrm{~m} / \mathrm{c}, \gamma \approx 6 \times 10^{-9} \mathrm{~m}^{-1}, \sigma \approx 7 \times 10^{-9} \mathrm{~m}^{-1}$. We make calculations for $k=2 \times 10^{7} \mathrm{~m}^{-1}$.

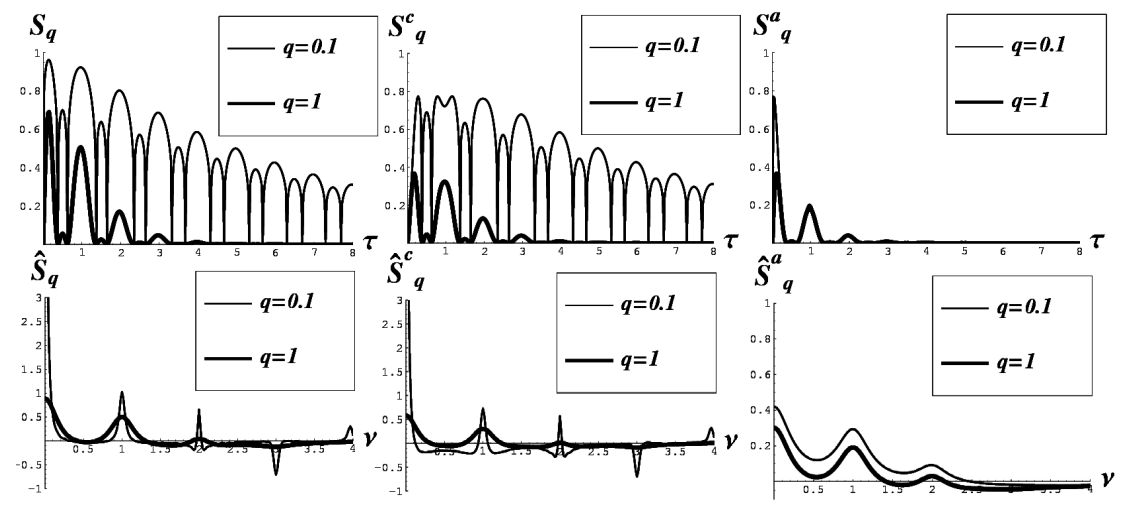

Fig. 5. Plots of entropies (13) for $q=0.1$ and (12) $(q=1)$ and their spectrum for the Landau-Placzek TCF. Here we use the following notations: $S_{q}=S_{q}^{0}, S_{q}^{\mathrm{c}}=S_{q}^{0 \mathrm{c}}$, $S_{q}^{\mathrm{a}}=S_{q}^{0 \mathrm{a}}$, and $\widehat{S}_{q}=\widehat{S}_{q}^{0}, \widehat{S}_{q}^{\mathrm{c}}=\widehat{S}_{q}^{0 \mathrm{c}}, \widehat{S}_{q}^{\mathrm{a}}=\widehat{S}_{q}^{0 \mathrm{a}}$.

In Fig. 5 we reproduce time and frequency dependencies entropies defined before for two values of parameter $q=1,0.1$. There is periodicity over $\tau$ with unit period which gives the appearance specific peaks in the frequency spectrum (the Brillouin doublet). We observe the same picture as in previous section. Decreasing the parameter of non-extensivity $q$ leads to increasing small peaks in entropies $S_{q n}$ and $S_{q \mathrm{~cm}}$, whereas quantity $S_{q \mathrm{am}}$ is changed insufficiently. The frequency 
spectrum entropies $S_{q n}$ and $S_{q c m_{n}}$ becomes more sharp. The shape of frequency spectrum $S_{q a m_{n}}$, in fact, does not change.

The non-Markovity parameter of this system has been calculated earlier in Refs. [24, 17]. It was shown that in hydrodynamical limit $k \rightarrow 0$ the spectrum of non-Markovity parameter has a form of alternating Markovian and non-Markovian levels.

\section{Ideal gas}

Let us consider the Fourier transformation of the fluctuation of the particle number density of a system

$$
\delta \rho_{k}(t)=\frac{1}{V} \sum_{l=1}^{N} \exp \left(\mathrm{i} \boldsymbol{k} \boldsymbol{r}_{l}\right)-\frac{N}{V} \delta_{k, 0}
$$

For this case the initial TCF is calculated exactly and it has the following form [22]:

$$
M_{0}(t)=\frac{\left\langle\delta \rho_{k}(t) \delta \rho_{k}(0)^{*}\right\rangle}{\left\langle\left|\delta \rho_{k}(0)\right|^{2}\right\rangle}=\mathrm{e}^{-t^{2} / t_{r}^{2}}, \quad t_{r}^{2}=2 m / k^{2} T .
$$

For this variable all the frequencies $\omega_{0}^{(n)}$ are equal to zero. The main relaxation frequencies in Eq. (1) have the simple form

$$
\Omega_{n}^{2}=n \Omega_{1}^{2}, \quad \Omega_{1}^{2}=k^{2} T / m
$$

Let us rescale time $t \rightarrow \tau=t \Omega_{1}$, and the Laplace transformation parameter $s \rightarrow c=s / \Omega_{1}$, and the Laplace images $\widetilde{M}_{n}(s) \rightarrow \widetilde{m}_{n}(s)=\Omega_{1} \widetilde{M}_{n}(s)$. In this case the hierarchy (1) has the following form:

$$
\widetilde{m}_{n+1}(c)=\frac{1}{n+1}\left[\frac{1}{\widetilde{m}_{n}(c)}-c\right]
$$

The Laplace image $\widetilde{m}_{0}(c)$ may be found in close form

$$
\widetilde{m}_{0}(c)=\mathrm{e}^{\frac{c^{2}}{2}} \sqrt{\frac{\pi}{2}} \operatorname{erfc}\left(\frac{c}{\sqrt{2}}\right),
$$

where $\operatorname{erfc}(x)=1-\operatorname{erf}(x)$ - additional probability integral.

The inverse transformation may be represented in the following form:

$$
M_{n}(\tau)=\frac{1}{2 \pi \mathrm{i}} \int_{\sigma-\mathrm{i} \infty}^{\sigma+\mathrm{i} \infty} \mathrm{e}^{c \tau} \widetilde{m}_{n}(c) \mathrm{d} c
$$

where $\sigma$ is greater than real part of zeros of $\widetilde{m}_{n}(c)$. By using the expression (23) we may set $\sigma=0$ and by changing $c \rightarrow \mathrm{i} x$ we obtain

$$
M_{n}(\tau)=\frac{1}{2 \pi} \int_{-\infty}^{+\infty} \mathrm{e}^{\mathrm{i} c x} \widetilde{m}_{n}(\mathrm{i} x) \mathrm{d} x,
$$

or in manifest form

$$
M_{1}(\tau)=\frac{1}{2 \pi} \int_{-\infty}^{+\infty} \mathrm{e}^{\mathrm{i} \tau x}\left[\frac{1}{\widetilde{m}_{0}(\mathrm{i} x)}-\mathrm{i} x\right] \mathrm{d} x,
$$




$$
M_{2}(\tau)=\frac{1}{4 \pi} \int_{-\infty}^{+\infty} \mathrm{e}^{\mathrm{i} \tau x}\left[\frac{1}{\frac{1}{\widetilde{m}_{0}(\mathrm{i} x)}-\mathrm{i} x}-\mathrm{i} x\right] \mathrm{d} x .
$$

We will calculate these formulae numerically. The first three memory functions and their spectrum are plotted in Fig. 6. In Fig. 7 we reproduce the plot of the entropy $S^{n}$ and their frequency spectrum for $n=0,1,2$.
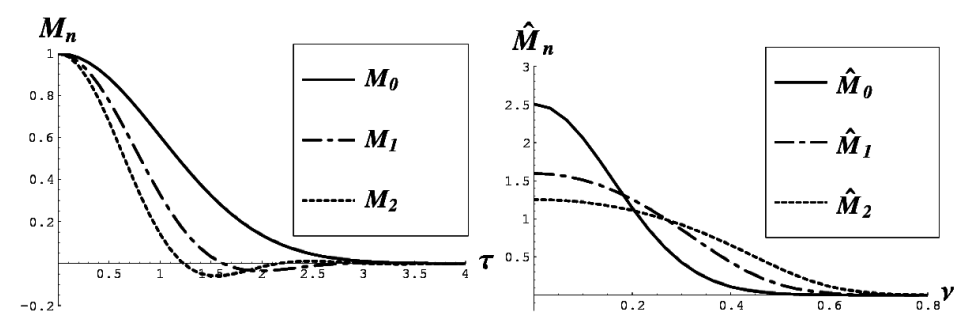

Fig. 6. The first normalized memory functions $M_{n}(\tau)$ and their frequency spectrum $\widehat{M}_{n}(\nu)$ for ideal gas.

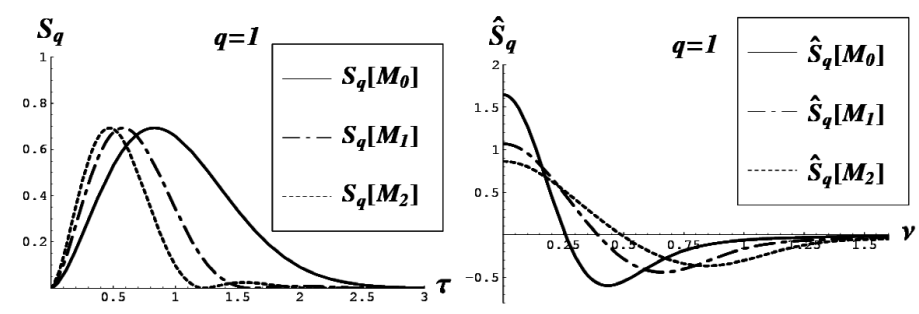

Fig. 7. The plot of the time dependence of entropy and its frequency spectrum for different relaxation levels $n=0,1,2$ for ideal gas.

It is not difficult to show that the entropy $S^{n}$ (12) amounts to maximum value, $\ln 2$, at the point $\left|M_{n}\right|^{2}=\frac{1}{2}$. For $n=0$ the position of maximum is at the point $\tau=\sqrt{\ln 2} \approx 0.832$. The greater $n$, the smaller time of maximum. The relaxation times $\tau_{0} \approx 1.6453, \tau_{1}=1.0688, \tau_{2}=0.8630$. The parameter of non-Markovity $[15,16]$ :

$$
\epsilon_{n}=\tau_{n} / \tau_{n+1}
$$

has the following values: $\epsilon_{0} \approx 1.54, \epsilon_{1} \approx 1.24$. Let us note that these values are very close to that calculated in paper [17] directly for TCFs, $\epsilon_{0} \approx 1.57, \epsilon_{1} \approx 1.27$.

In Fig. 8 we give the plots of entropy $S_{q}^{n}$ and their spectra for $q=1 / 2$ and $q=3 / 2$. In order to show what happens if we will vary the value of $q$ we reproduce in Fig. 9 the entropy $S_{q}^{n}$ and its spectrum for $n=2$ and for $q=0.5,1,1.5$. To show the dependence $S_{q}^{n}$ for more wide range of $q$ in Fig. 10 we give entropies for more wide range of $q$. 


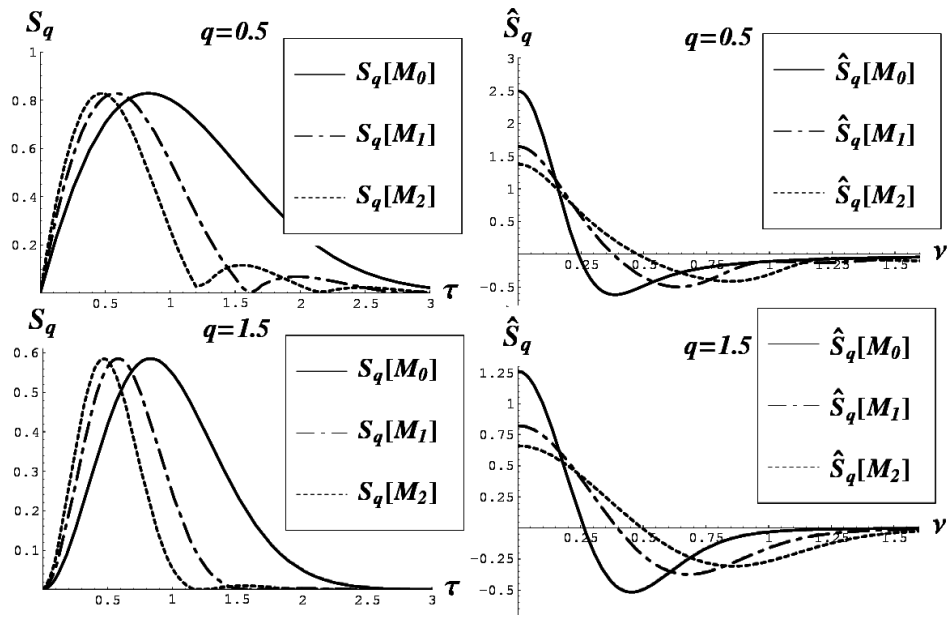

Fig. 8. The plots of time dependent entropy $S_{q}^{n}$ and their frequency spectra for $q=1 / 2$ and $q=3 / 2$.

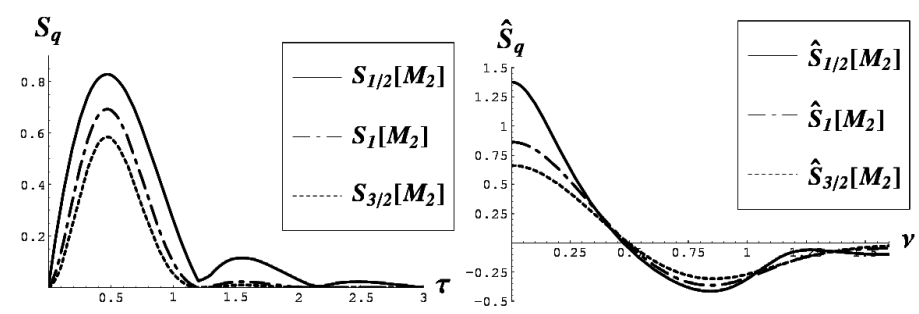

Fig. 9. The time dependent entropy $S_{q}^{n}$ and their frequency spectrum for $n=2$ and for $q=0.5,1,1.5$.

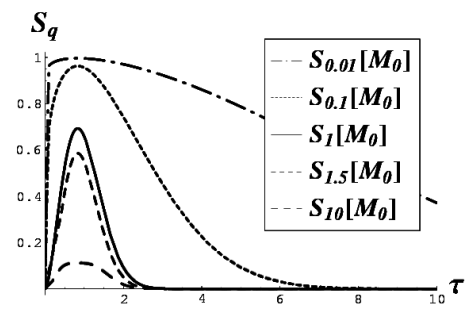

Fig. 10. The time dependence of the entropy $S_{q}^{n}$ and its frequency spectrum for six values $q=0.001,0.1,0.5,1,1.5,10$ and for $n=0$. One observes that the increasing Tsallis parameter $q$ leads to trampling a quantity of $S_{q}$ in domain of short time. Due to this fact one can make sufficient amplification in domain of short time owing to variation parameter $q$. 


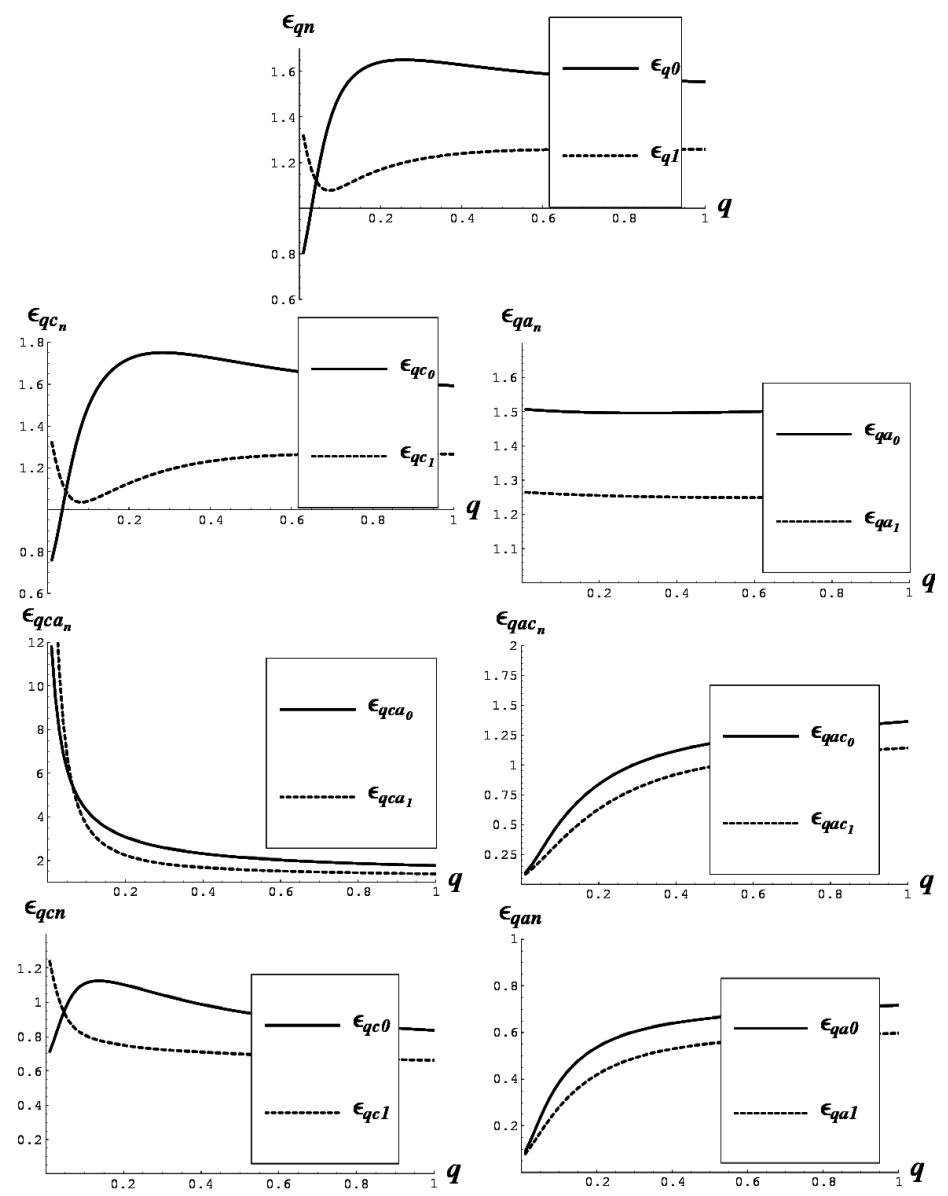

Fig. 11. The spectrum of all parameters of non-Markovity as function of $q$. The influence of parameter $q$ is most effective for small values of $q \ll 1$. In all cases we observe strong non-Markovity and clearly marked effect of statistical memory. The only case where we may sufficiently increase the non-Markovity properties by decreasing parameter $q \rightarrow 0$ is cross term $c a$ (see Eq. (24b)). For small enough $q \ll 1$ the non-Markovity parameter $\epsilon_{q c a_{n}}$ may reach great value $\epsilon_{q c a_{n}} \gg 1$.

By using these three quantities we define seven different spectra of non-Markovity parameter:

$$
\begin{aligned}
& \epsilon_{q n}=\frac{\tau_{q n}}{\tau_{q n+1}}, \quad \epsilon_{q c_{n}}=\frac{\tau_{q c n}}{\tau_{q c n+1}}, \quad \epsilon_{q a_{n}}=\frac{\tau_{q a n}}{\tau_{q a n+1}}, \\
& \epsilon_{q c a_{n}}=\frac{\tau_{q c n}}{\tau_{q a n+1}}, \quad \epsilon_{q a c_{n}}=\frac{\tau_{q a n}}{\tau_{q c n+1}}, \quad \epsilon_{q c_{n}}=\frac{\tau_{q c_{n}}}{\tau_{q n+1}}, \quad \epsilon_{q a_{n}}=\frac{\tau_{q a n}}{\tau_{q n+1}} .
\end{aligned}
$$

The plots of all quantities are shown in Fig. 11. As expected, at the beginning more interesting situations are possible for small values of $q$. For great value of $q$ all lines tend to constants. 


\section{Conclusion}

Let us summarize our results. We considered the dynamic Tsallis entropy and applied it for model systems. In accordance with Refs. [8-10] the square of TCF is regarded as probability of dynamic state. We used the time dependent entropies as well as their frequency spectra. We considered four model TCFs which, nevertheless, have physical sense. The first and second models describe the motion of Brownian oscillator without and with noise. The noise is modelled by generator of random numbers. The third model is the Landau-Placzek model for TCF of particle density fluctuations in hydrodynamic limit. The last model is ideal gas, the relaxation of particle density fluctuations.

In all models we considered different values of parameter of non-extensivity $q$. All entropies have the same structure zeros and extrema as square of TCF. The magnitude of extrema sufficiently depends on value of $q$. Small values of $q$ act as non-linear magnifier: the smaller magnitude of entropy the greater magnification. Great values of $q$ act in opposite way as demagnified lens. Concerning the frequency spectra of entropies we observe that for small values of $q$ the peaks become more sharp and larger. For systems with noise this property works better. It is possible to reveal peaks even if they are lost in noise.

For ideal gas we additionally calculated the spectrum of non-Markovity parameter by using different definition of relaxation time. By using three kinds of information Tsallis entropy we defined seven kinds of spectrum of non-Markovity parameter. We observe that all of these parameters (except $\epsilon_{q c a_{n}}$ ) are close to unity. It means that the ideal gas remains non-Markovian system for arbitrary value of $q$. It is in qualitative agreement with Ref. [25]. The variation $q$ from unity does not mean the appearance of new interactions. The system becomes ideal and non-Markovian from this point of view.

Our analysis allows us to conclude that the use of dynamic Tsallis entropy extends essentially possibilities of the stochastic description of model physical systems. Advantage of application of DTE is that it allows to strengthen or suppress fluctuations either in low-frequency or in high-frequency areas of a spectrum. Similar supervision opens appreciable prospects in the field of the study of real complex systems of wildlife where dynamic states of physiological and pathological systems are very important.

\section{Acknowledgments}

This work was supported in part by the Russian Foundation for Basic Research grants No. 05-02-16639, 03-02-96250 and by the Russian Humanitarian Scientific Foundation grant No. 03-06-00218a. 


\section{References}

[1] C. Beck, F. Schlogl, Thermodynamics of Chaotic Systems, Cambridge University Press, Cambridge 1993.

[2] S.A. Kaufman, The Origins of Order - Self-Organization and Selection in Evolution, Oxford University Press, Oxford 1993.

[3] I. Goychuk, P. Hanggi, Phys. Rev. E 61, 4272 (2000).

[4] Yu.L. Klimontovich, Phys. Scr. 58, 549 (1998).

[5] V. Latora, M. Baranger, A. Rapisarda, C. Tsallis, Phys. Lett. A 273, 97 (2000).

[6] D.J. Scapino, M. Sears, R.A. Ferel, Phys. Rev. B 6, 3409 (1972).

[7] C. Tsallis, Braz. J. Phys. 29, 1 (1999).

[8] R.M. Yulmetyev, M.Ya. Kleiner, Nonlinear Phenomena in Complex Systems 1, 80 (1998).

[9] R.M. Yulmetyev, F.M. Gafarov, Physica A 273, 416 (1999); R.M. Yulmetyev, F.M. Gafarov, Physica A 274, 381 (1999).

[10] R.M. Yulmetyev, F.M. Gafarov, D.G. Yulmetyeva, N.A. Emeljanova, Physica A 303, 427 (2002).

[11] A. Katok, B. Hasselblatt, Introduction to the Modern Theory of Dynamical Systems, Cambridge University Press, Cambridge 1995.

[12] C. Tsallis, J. Stat. Phys. 52, 479 (1988).

[13] A. Aczél, Z. Daróczy, On Measures of Information and Their Characterizations, Academic Press, New York 1975.

[14] R.M. Yulmetyev, N.A. Emeljanova, F.M. Gafarov, Physica A 341, 649 (2004).

[15] V.Yu. Shurygin, R.M. Yulmetyev, V.V. Vorobjev, Phys. Lett. A 148, 199 (1990).

[16] V.Yu. Shurygin, R.M. Yulmetyev, Zh. Eksp. Teor. Fiz. 99, 144 (1991) [Sov. Phys.-JETP 72, 80 (1991)].

[17] R.M. Yulmetyev, N.R. Khusnutdinov, J. Phys. A 27, 5363 (1994).

[18] H. Mori, Prog. Theor. Phys. 33, 423 (1965); 34, 765 (1965).

[19] R. Zwanzig, Phys. Rev. 124, 1338 (1961).

[20] M. Reed, B. Simon, Methods of Modern Mathematical Physics, Academic, New York 1972.

[21] R. Zwanzig, Ann. Rev. Phys. Chem. 16, 67 (1965).

[22] P. Résibois, M. De Leener, Classical Kinetic Theory of Fluids, Wiley, New York 1977.

[23] N.B. Vargaftik, Handbook of Physical Properties of Liquids and Gases: Pure Substances and Mixtures, 2nd ed., Hemisphere Pub, Washington 1983.

[24] N.R. Khusnutdinov, R.M. Yulmetyev 1995, Teor. Matem. Fiz. 105, 292 (1995) [Theor. Math. Phys. 105, 1426 (1995)].

[25] S. Abe, Physica A 269, 403 (1999). 